\title{
AFECÇÕES CIRÚRGICAS INTRACRANIANAS NA INFÂNCIA E ADOLESCENCIA. ANÁLISE DE 103 CASOS. I: DISTRIBUIÇÃO EM RELAÇÃO À IDADE, SEDE E NATUREZA
}

\author{
Aiorsio Majtos p'imfinta \\ Aronso Si:TTE JR. \\ Rosa Helena Longo
}

A importância da correlação dos elementos idade, sede e natureza no estudo dos tumores intracranianos ficou demonstrada após a revisão de material cirúrgico numeroso. A concentração do material de tumores intracranianos estudados clínico-histològicamente só se tornou possível com o aparecimento da especialização e organização de centros neurocirúrgicos.

Os estudos de Cushing e de Bailey e col. ${ }^{1}$ sôbre a patologia dos tumores já mostravam de modo documentado a prevalência de certos tumores conforme os grupos de idade dos pacientes. Foi evidenciada a predileção do meduloblasioma para a infância, como fator idade, e no vérmis, como fator sede. Cushing e Bailey, Foerster e Zülch evidenciaram a dominância dos gliomas entre os tumores intracranianos. No entanto, os trabalhos iniciais (Starr, Garland e Armelaje, Fiori, cit. por Bailey ${ }^{1}$ ) mostravam a predominância dos tuberculomas, fato que os trabalhos mais recentes não confirmam. .

Vemos na literatura que, entre os tumores da infância e adolescência, dominavam, quanto à natureza, os gliomas, e, entre êles, o meduloblastoma. Considerando o fator sede puderam ser demonstrados vários dados de interêsse para o clínico: assim, por exemplo, a síndrome da linha média cerebelar na infância está em relação com o meduloblastoma e a síndrome do ângulo pontocerebelar no adulto, em relação com o neurinoma do acústico.

Portanto, os fatôręs idade, sede e natureza nas afecções cirúrgicas in. tracranianas podem fornecer ao clínico elementos de diagnóstico e prognóstico. Assim, resolvemos rever nosso material de pacientes abaixo de 20 anos, idade limite nas séries analisadas no Serviço de Tönnis ${ }^{3}$.

Trabalho apresentado ao Departamento de Neuro-Psiquiatria da Associação Paulista de Medicina, em 5 dezembro 1953. 
Tendo em vista o interêsse clínico, estudamos não sòmente as neoformações, mas tôdas as afecções cirúrgicas verificadas. Excluímos os casos em que a ação cirúrgica foi de tipo funcional, como nas hidrocefalias e leucotomias.

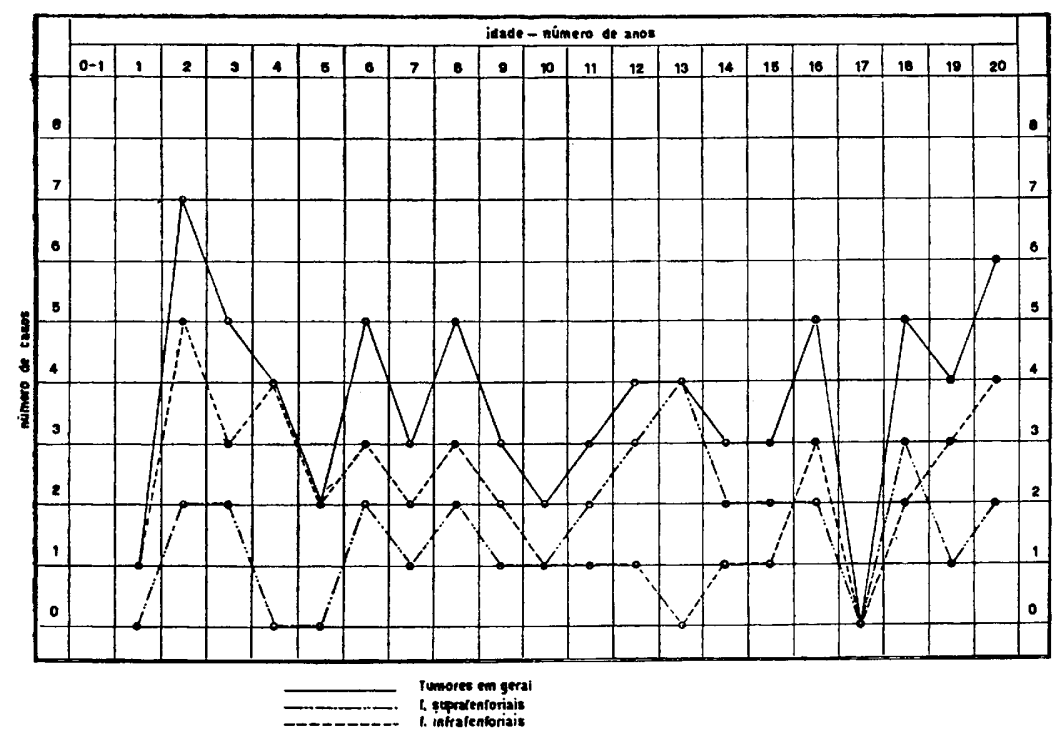

Gráfico 1 -- Distribuição de acôrdo com idade e sede infra ou supratentorial.

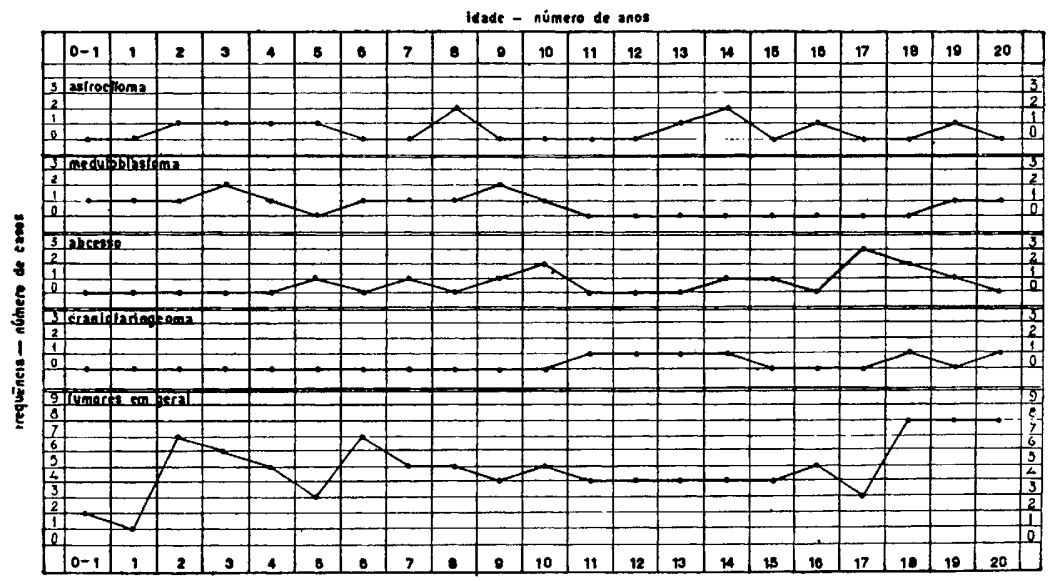

Gráfico 2 - Distribuição de acôrdo com idade e natureza mais freqüente. 
Consideramos os fatôres natureza (quadros 1 e 2) e sede (quadro 3) seguindo os esquemas dos trabalhos de Zülch ${ }^{5}$. Além do mais, apresentamos a distribuição do material em relação à idade considerando o número global, a sede infra ou supratentorial (gráfico 1) e a distribuição das lesões mais freqüentes (gráfico 2).

\section{RESUMO E CONCLUSÖES}

Quanto à natureza (quadros 1 e 2) -- 1) A classificação histológica traz em si fatôres de êrro devido à existência, na mesma peça, de zonas de constituição celular diferente, e à diversa orientação seguida pelos patologistas. 2) Os gliomas constituem o grupo dominante, sendo que: $a$. o glioblastoma, tumor próprio da idade adulta, apareceu tanto em situação supratentorial como infratentorial; $b$. o astrocitoma foi dominante no hemisfério cerebelar; $c$. o meduloblastoma apareceu como lesão de fossa posterior, nem sempre na linha média e excepcionalmente como lesão de hemisfério cerebral; $d$. o ependimoma só apareceu no $4^{\circ}$ ventrículo, não aparecendo no hemisfério cerebral, como mostrou Tönnis (temos um caso de neuroepitelioma de hemisfério cerebral que alguns patologistas classificam como ependimoma); $e$. os papilomas foram os tumores intraventriculares. 3) Os meningeomas foram raros em nosso material. 4) 0 neurinoma foi excepcional. 5) Nos tumores da região hipofisária, não tivemos casos de adenoma, mas apenas craniofaringeomas e gliomas do nervo óptico. 6) $O$ hemangioblastoma, de freqüência desusada em nosso material geral, requerendo mesmo revisão de estudo histológico, não foi dominante nas duas primeiras décadas da vida. 7) Dos tumores malignos de início clínico no sistema nervoso dominou o reticulossarcoma. 8) Das afecções cirúrgicas não tumorais apareceram de preferência os hematomas, os abscessos e a cisticercose (em relação à cisticercose devemos dizer que foram relacionados apenas os casos verificados cirùrgicamente). Os tuberculomas foram raríssimos. $\mathrm{O}$ aneurisma arteriovenoso, lesão que começa com sintomas no início da $2^{\mathrm{a}}$ década, é diagnosticado geralmente mais tarde. Em nosso material alguns casos apareceram após a análise dêste grupo.

Quanto à sede (quadro 3) - 1) Em conjunto, não houve dominância nítida das lesões infratentoriais sôbre as supratentoriais, como é clússico na literatura médica. 2) Em relação ao grupo dos gliomas, a dominância infratentorial foi evidente. 3) Nos infratentoriais dominaram, como tipo maligno, o meduloblastoma e, como benigno, o astrocitoma. 4) 0 astrocitoma foi dominante nos hemisférios cerebelares, enquanto o meduloblas- 

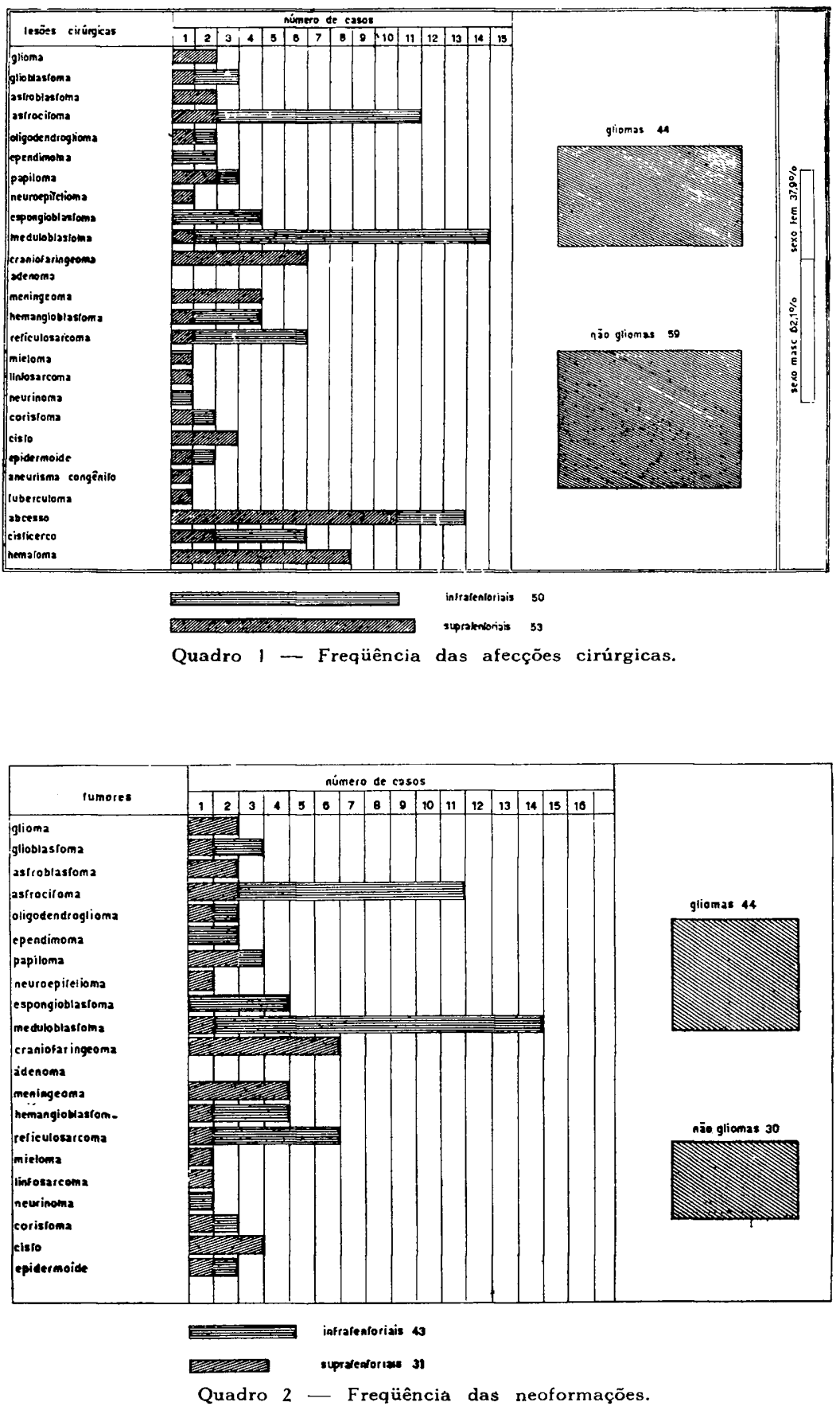


\begin{tabular}{|c|c|c|c|c|c|c|c|c|c|c|c|}
\hline \multirow[b]{4}{*}{ glioma } & \multicolumn{6}{|c|}{ suprarentorial } & \multicolumn{5}{|c|}{ infratenforial } \\
\hline & \multirow[t]{3}{*}{ k } & \multirow[t]{3}{*}{ fi-pak } & \multirow[t]{3}{*}{ p. } & \multirow[t]{3}{*}{$I-0$} & \multirow{3}{*}{ x.1. 3r. hipoit. } & \multirow{3}{*}{\begin{tabular}{|l|} 
noplice \\
$\bullet$
\end{tabular}} & \multirow[t]{3}{*}{$4 v$} & \multirow[t]{3}{*}{ guedulo } & \multicolumn{2}{|c|}{ cerebelo } & \multirow[t]{3}{*}{ Fronco } \\
\hline & & & & & & & & & vermis & memintorios & \\
\hline & & & & & & & & & & & \\
\hline glioblasioms & & & $\bullet$ & & & & & & & & $\bullet \bullet$ \\
\hline aetrowastoms & $\bullet$ & & & - & & & & & & & \\
\hline astrocilome & $\bullet$ & & $\bullet$ & & & & $\bullet$ & & - & $:: 8$ & \\
\hline oligodendreglioma & $\bullet$ & & & & & & & & - & & \\
\hline ependimoma & & & & & & & $\bullet$ & & & & \\
\hline papiloms & & & & & $\bullet \bullet$ & & $\bullet$ & & & & \\
\hline neuroepifeliome & & & $\bullet$ & & & & & & & & \\
\hline \multicolumn{12}{|l|}{ espongioblationa } \\
\hline meduloblasloma & & & $\bullet$ & & & & & & $::: \div$ & $8:$. & $\bullet$ \\
\hline refleubsarcems & $\bullet$ & & & & & & & & & $8: 6$ & \\
\hline \multicolumn{12}{|l|}{ ermiotaringeoma } \\
\hline \multicolumn{12}{|l|}{ adenoma } \\
\hline meningeoma & $\bullet \bullet$ & & & & $\bullet$ & & & & & & \\
\hline hernangioblastome & $\bullet$ & & & & & & $\bullet$ & & $\bullet$ & $\bullet$ & \\
\hline mieloma & & & & & $\bullet$ & & & & & & \\
\hline lintosarcoma & $\bullet$ & & & & & & & & & & \\
\hline neurinoms & & & & & & & & & & $\bullet$ & \\
\hline corisfoma & $\bullet$ & & & & & & & - & & & \\
\hline cisto & $\bullet$ & & $\bullet$ & & $\bullet$ & & & & & & \\
\hline epidermoide & & & & & $\bullet$ & & & & & & $\bullet$ \\
\hline a neurisma congếito & $\bullet$ & & & & & & & & & & \\
\hline ruberculoma & & & & & $\bullet$ & & & & & & \\
\hline abcesso &.$<$. & $::$ & 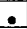 & 6 & & & & & & . & \\
\hline cisticerco & - $\bullet$ & & & & & & - $\bullet$ & $\bullet$ & & & \\
\hline hematoms. & $8: 8$ & - & & $\bullet$ & & & & & & & \\
\hline
\end{tabular}

Quadro 3 - Distribuição das afecções cirúrgicas de acôrdo com a sede.

toma apareceu tanto na linha média como nos hemisférios cerebelares. 5) $\mathrm{Na}$ região hipofisária (optoquiasmática) dominaram os craniofaringeomas e apareceram os gliomas do nervo óptico, não se registrando casos de adenoma.

Como conclusões gerais: 1) A correlação dos fatôres idade, scde e natureza fornece elementos para o diagnóstico e prognóstico dos casos de afecções cirúrgicas intracranianas; 2) Em nosso material não houve a clássica dominância das lesões infratentoriais sôbre as supratentoriais, mas as demais distribuições de sede e natureza estão de acôrdo com os dados da literatura médica; 3) 0 meduloblastoma tanto apareceu nos hemisférios cerebelares como na linha média, sua sede preferencial de acôrdo com a literatura.

\section{SUMMARY}

In patients with surgical intracranial lesions, correlation of age, localization and type is very important to diagnosis and prognosis. In our material we have not found the classical predominancy of infratentorial 
lesions over the supratentorial; the localization and type of lesions agree with the literature. Medulloblastomata are so common in the cerebellar hemispheres as in the vermis, the latter being the most common site according to the literature.

\section{BIBLIOGRAFIA}

1. Bailey, P.; Buchanan, D.; Bucy, P. - Intracranial 'Tumors in Infancy and Childhood. University of Chicago Press, Chicago, 1939. 2. Cuneo, H.; Rand, C. Brain 'Tumors of Childhood. Ch. C. 'Thomas, Springfield, 1952. 3. Tönnis, W. Hirngeschwülste im Kindesalter. Kinderärztl. Praxis, 8:97-108, 1937. 4. Zülch, K. J. - $a$ ) Die Hirngeschwülste im Kindesalter. Ltschr. f. d. ges. Neurol. u. Psychiat, 161:183-188, 1930; b) Die Hirngeschwülste in Jugendsalter. Zentralbl. f. NeuroChir., 5:238, 1940. 